Fliegen mit einer Klappe geschlagen werden“. Denn die aus Mastzellen und Eosinophilen sezernierten CysteinylLeukotriene sind am entzündlichen Grundprozess als auch an der Bronchokonstriktion beteiligt: Sie wirken hochgradig chemotaktisch auf Eosinophile und stark bronchokonstriktorisch.

Ihre Wirkung entfalten die Cysteinyl-Leukotriene über die an den Bronchien lokalisierten Cys-LT1Rezeptoren. Eine Blockade dieser Rezeptoren durch Leukotrienantagonisten wie Montelukast (Singulair ${ }^{\circledR}$, Singulair ${ }^{\circledR}$ junior $5 \mathrm{mg}$ ) verhindert die Asthma auslösenden Effekte der Mediatoren. Aufgrund dieser Wirkqualitäten wird Montelukast heute als Controller mit zusätzlichen bronchodilatierenden Eigenschaften eingestuft.

Zugelassen ist Montelukast zum einen für die Behandlung des Belastungsasthmas, wobei die fehlende Toleranzentwicklung ein Pluspunkt z.B. gegenüber dem langwirksamen Beta2Mimetikum Salmeterol darstellt, sagte Virchow. Das zweite offiziell zugelassene Indikationsgebiet ist die Kombinationstherapie bei Patienten mit leichtem bis mittelschwerem Asthma. In kontrollierten Studien ergab sich durch die zusätzliche orale Gabe von 10 bzw. 5 mg Montelukast (Erwachsene bzw. Kinder) einmal täglich eine deutliche Verbesserung der FEV1-Werte, eine Reduktion der erforderlichen Kortikoiddosis und des Beta2-Mimetika-Verbrauchs sowie eine Senkung der Exazerbationshäufigkeit.

\section{Kräftemessen mit Kortikoiden}

Doch liegen nach den Worten Virchows mittlerweile mehr als nur Hinweise dafür vor, dass der Leukotrienantagonist auch in der Monotherapie wirksam ist wenngleich auch nicht in der Wirkstärke wie Kortikoide. So ergab eine Studie mit 895 Asthmapatienten (Malmstrom $\mathrm{K}$ et al. Ann Intern Med 1999; 130: 487-95) insgesamt eine vergleichbare Wirkung von Beclometason und Montelukast. Gegenüber Plazebo erzielte der Leukotrienantagonist jedoch ebenfalls eine signifikant überlegene Wirkung auf alle relevanten Asthmaparameter.

Sogar vergleichbar waren die Wirkungen beider Verummedikationen in der Reduktion akuter Exazerbationen, „einem sehr wichtigen klinischen Parameter", so Virchow. Und in einem Punkt zeigte sich Montelukast dem Kortikoid als überlegen: Der Wirkeintritt erfolgte wesentlich rascher.

Speziell bei Kindern fällt die gute Verträglichkeit und vor allem die nur einmal tägliche orale Gabe von Montelukast besonders ins Gewicht, sagte H.G. Bresser (Bielefeld), die Compliance ist gegenüber inhalativ einzunehmenden Medikamenten sehr viel besser.

Auch Kinder im Alter zwischen zwei und fünf Jahren profitieren von der gut verträglichen Therapie, so die Er- gebnisse neuerer Studien. In den USA ist das Medikament in einer Dosierung von $4 \mathrm{mg} / \mathrm{d}$ daher bereits für die Behandlung von Asthma bei Kindern ab zwei Jahren zugelassen. Bei uns gilt noch die Grenze von sechs Jahren. „Mit einer offiziellen Absenkung der Altersgrenze ist aber auch in Deutschland schon bald zu rechnen", so Bresser. $\quad b k$

\section{Virchow Ch, Bresser H-G}

Pneumologen-Workshop „Asthma in real Life - Stimmen die Therapiekonzepte noch?", veranstaltet von der MSD Sharp \& Dohme $\mathrm{GmbH}$, Dresden 2000.

\title{
Erstes TÜV-Prüfsiegel für ein luftdurchlässiges Encasing
}

\section{Als bisher einziger unbeschichteter und damit luft- und wasser- dampfdurchlässiger Encasing für das Bett des Hausstaubmilben- allergikers hat Allergocover ${ }^{\circledR}$ das TÜV-Zertifikat „Für Allergiker geeignet" erhalten.}

V

on bisher mehr als zehn untersuchten Produkten konnten lediglich vier die Anforderungen der freiwilligen TÜV-Testung erfüllen und erhielten das Prüfsiegel „Für Allergiker geeignet“. Das Besondere an Allergocover ${ }^{\circledR}$ : Er ist bislang der einzige unbeschichtete und luftdurchlässige milbendichte Zwischenbezug, der dieses Qualitätssiegel tragen darf.

\section{Der Alltagstest}

Die Prüfkriterien und -verfahren für diese Testung wurden in den letzten Jahren u.a. in enger Kooperation mit Allergologen und unabhängigen technischen Instituten entwickelt. Unter realitätsnahen Bedingungen lag der Hausstauballergengehalt in der Raumluft bei Verwendung von Allergocover ${ }^{\circledR}$ um ein 50-faches niedriger als der Schwellenwert, der als Auslöser von Beschwerden bei Allergikern bzw. sensibilisierten Menschen gilt. Auch gegenüber häufig im Inneren der Betten verborgenen Schimmelpilzen leistet Allergocover ${ }^{\circledR}$ Schutz: Das luftdurchlässige, unbeschichtete Spezialgewebe hält selbst nach Monaten einer Durchwachsung mit Schimmelpilzhyphen stand.

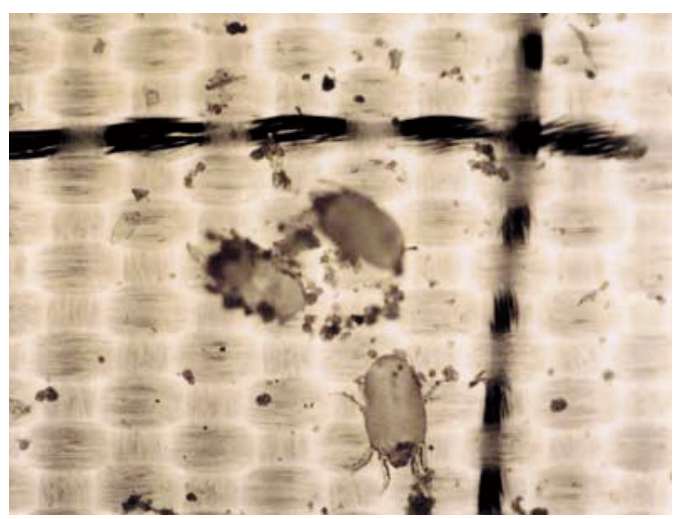

Schwierige Gradwanderung: Encasings müssen Luft- und Wasser- durchlässig, aber gleichzeitig Milbenallergen-dicht sein.

Gegenüber beschichteten oder laminierten Produkten bietet Allergocover, den Vorteil einer deutlich höheren Luft- und Wasserdampfdurchlässigkeit. Damit zeichnet sich Allergocover ${ }^{\circledR}$ neben der TÜV-geprüften Sicherheit durch einen hervorragenden Schlafkomfort aus und führt daher zu einer guten Patientencompliance.

Nach Informationen der Allergopharma Joachim Ganzer KG, Reinbek. 